\title{
Experience Internet of Things by the Gateway of Smart Home Spectrum
}

\author{
Devika Soni \\ ASET, Amity University Uttar Pradesh, Noida (INDIA) \\ Email: devikagrand2@gmail.com \\ Received 06.02.2021, Received in revised form 19.03.2021, Accepted 27.03.2021
}

\begin{abstract}
The IOT is the large-scale diversification of internet association connectivity to perpetuity relating the surfeit of physical devices and day to day objects revolving hardware's, internet connectivity, Arduino coding, WIFI modules, sensors, monitoring and control system, etc. Internet sets out as a means for communication for these devices and volleyed accordingly. The project is riveted on the automatic lightening system with the "ON" and "OFF" of lights spectrum, with the sensors (PIR) that detects the human existence in the domestic and delivers information about the number of folks in the room , moreover the user can choose the intensity of the light itself , also the database is created for the same . Also! If the human leave. On barging past consciously, it'll be taken care of to switch off the lights. The main aim is to create awareness for the user to keep in account the usage and consumption of energy. To lay emphasize on the LED bulbs in smart home automation. Embracing the latest era of connectivity in the realm of technology.
\end{abstract}

Keywords- Arduino ide, Pir sensor, NODEMCU WiFi module, keypad, php My Admin.

\section{INTRODUCTION}

Everyday things, the IOT is an extensively massive expansion of devices that are internet connected. Thus, are supervised and foreseen by the control of sensors in and around us. The internet has evolved and blossomed and is crafted as a fabric in our daily life in one way or the other. On a sketchier note, this spectrum is not about connecting people but it's about connecting things [1].

Things share their experiences with other things. The fundamentals encircle to cumulation of sensing, communicating, touching, controlling. The internet is the most important hand transform ante of technologies ever invented. The technologies inherited by the IOT like the capability to sense, communicate, acquire data in addition, to fabricate multiple process and system. The 5 roots of internet of things: invent, build, optimise, operate, innovate, enjoy. Create, construct, sanguine, make way, rooted innovation and technology profoundly and aspiring and inspiring the universe to perpetuity and beyond.

\section{A. Smart home automation}

"SMART HOMES" it can anticipate our needs. A smart home is autonomous, first and foremost it does the right thing at the right time without, the human intervention. For example, adjusting the smart thermostat as we walk, is the A1 devices adopted. The smart door locks, the door on the door can rekey themselves smart termous can be turned off, smart heater can be shut automatically. Everything mentioned above is a tangible consumption for the members of the house. Consumers are using them for positively bagged up impact in their life's. A smart home is aware it has 200 senses in it altogether. Roughly, of all kinds. The house is the user absolute interface. We emerge an internet connected devices to remotely monitor and manage plethora of systems and applications in our homes. An angle to the smart home's idea or innovation serves as a recipe, a model, a fuel to replicate around the country.

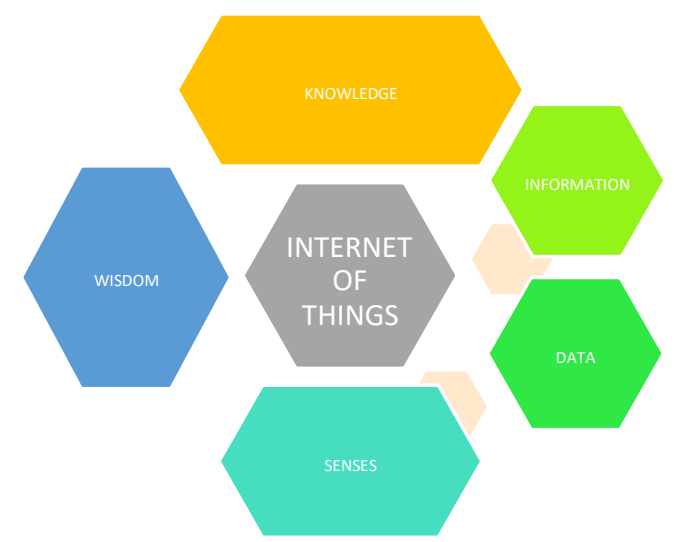

Fig.1 The above figure displays 5 components which bring about the technology of IOT.

\section{B. Automatic lightening system}

Revolving around the opening and closing of lights in a room, automatically within a smart home. Confounded with electricity that's one among the prominent and immensely crucial resources thus, must be utilized accordingly. . For instance, as part our fabric when the regimen gets triggered and we tend to forget the closing and opening of lights while exiting the room. Well! The technology takes over and leaves the tension at bay. In the backhand the devices and sensors workup on it [3]. Automated 
lights deliver a sense of belongingness to the domestic place, keep home illuminating at all hours of the day. Basically, a lightening control system is nothing but a brain power network with the interrelation of input and output, along with the presence of the controlling unit.

\section{PROPOSED METHODOLOGY}

Controlling Lightening as per the will and wish of the user is the prime focus with the ultimate agenda of providing an efficient and effective utilization of energy bagged with saving and consumption pattern. Stepping deeper, Automatic Lights using, a massively used internet of things platform which provides the cheapest open source to work upon NODEMCU confound Arduino ide coding and PIR Sensor (turn ON and OFF by detecting the presence of a human in their domestic). With the proposed model such sort of Automatic Room Lights can be device in the home garages, staircases, bathrooms, etc. Moreover, where illuminance isn't required, it acts accordingly and vice versa. Also! With the fundamentals and basis of automatic room light control system, the mankind should be least bothered existing in the realm of technology, When, no presence of human detected, the lights would shut automatically. The second part of the plot is to note the intensity of the light, the user participation plays a preposterously crucial role here, in accordance to it, and the database is noted as per the desired intensity chosen by the user via a 9-digit modernized keypad. Further which, SQL (Structured query language) has greater implication in the prologue such as in order to access, refine and restructure database inclusive of 5 attributes: S.No, name, IR intensity, Date, Start time, End time. Thus, the database is prepared via SQL fundamentals, it'll showcase the variation and the usage of the light intensity per day and an amalgamation of the month and year in total. For instance, the light intensity set by the user on $1^{\text {st }}$ of January 2020 at $12 \mathrm{AM}$ is 6 and later on , the user switches the intensity to 4 at around 5:45 AM, the intensity stations itself at 4 . Later by $1 \mathrm{pm}$, again the user switches the intensity via the keypad at 7 . Moreover, the user dismisses and changes the intensity to 4 at $10 \mathrm{pm}$. lastly! The intensity changes to 3 at 12 am again. In a nutshell, the 24 hour cycle is noted and imprinted upon the system which can be accessed by the windows, MAC, Linux etc. Similarly, one month followed by the other that is, January, February, March...... December. Each respective month's cumulative database is prepared on the system. In stark contrast, to the light intensity and the usage will bring about an awareness in the consumers conscious mind. Hence, by the end of the year the consumer be meticulously riveted on the energy saved and consumed. The coding is done in embedded c programming language on Arduino ide. Here! I'd put forward the two strokes of my proposed model that is, hardware's and software:

- NODE-MCU: Serves as an open source internet of things platform, with embedded ESP 8266 WIFI module. Implication of it, connecting NODEMCU to the computer, via a usb micro B cable to the board so as to link the Arduino ide code work upon.

- ARDUINO IDE: In Arduino ide, the kind of programming languages that are used are C, $\mathrm{C}++$ etc. Here, basically, it complies the code, craftingly easy to grasp, it again is a userfriendly, can run simple low power programmes and can be powered and sustain for days.

- RELAY: A relay is nothing but an electrical switch that uses the basis of electromagnetics to move the switch from the "ON" to "OFF" position followed by no human intervention.

- JUMPER WIRES: Main usage is to simulate connections between items and Arduino's header pins installing a single package. Also, this will make a local server to connect all the things bagged with WIFI.

- SQL FUNDAMENTALS: Database means to pull out the data, we aspire and intend to see is done by SQL, it's a language which communicates with databases. Databases are

- Nothing but store the data in it. SQL stands for structured query language. Components are in Order to pull, edit, add information to a database, there SQL comes into action and it plays it role.

- PHPMYADMIN MYSQL and later on click on the admin button next to MySQL. Then, in the localhost tabular column underneath it write a name to database, mention the required number of column in the adjacent tabular column. Further exercise the fundamentals of SQL and begin. After the XAMPP, press on the apache and MySQL.

- XAMPP CONTROL PANEL V 3.2.4: It is a single installer that allows one to install the packages, once the package is installed, thereafter one can access to MySQL, php, proem. Thus, very easy to install and get a webserver running, by simply installing a single package. Also, this will make a local server to connect all the things bagged with Wi-Fi. 


\section{EXPERIMENTAL RESULT AND DISCUSSION}

A startling idea, which encircles the automatic lightening system "opening" and "closing" of lights, the PIR sensor at a certain distance from the main door is implanted. In fact two of them are planted with a tiny distance between them. Let me ignite with an illustrative interpretation, as though when a person enters will enter the domestic, the PIR sensor at a certain distance from it, will start upheaval on the presence of the human existence, LED: A light-emitting diode (LED) with raised output to turning "ON" of an LED, the Arduino needs to beep a HIGH.

The similar kind of process reciprocates with the folks, arrivals and departure. Stepping deeper into the roots, passing by PIR1+PIR2 would give an increment the people. The person count will enhance by 1 in the domestic. Whereas, reverse PIR2+PIR1 would be Riveting on the decrement in the people.

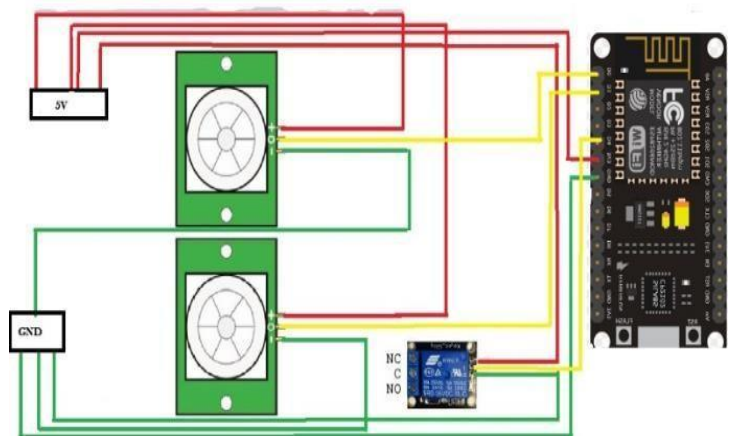

Fig.2 Diagrammatic representation of the proposed model

That is, the person count will descend by 1 in the domestic. Adhering to the topic, presence detected and triggering would give rise to the "ON" mode of light. Presence left undetected and no alarming would cause the "OFF" mode of the light. Well! The tip of iceberg is that, when the mode of light is "ON" it will be able reveal the status of the people in the domestic. In short, it will give the user the People status. Now moving further, to the second dimension, the fluorescence intensity. The opening and closing of the lights is a part of the first dimension of my project, in coherence to it, emanated from the first, dimension itself. Fluorescence intensity, the user with the exploring traits will have a 9 type-pad, that is, it will have a 0 9 digits. Thereby, 0 indicates low intensity, between 0-5 (strokes of lowness of intensity), 5 is moderate, 9 is the peak intensity, 5-9 (range of highness of the light intensity) the user is given utmost venture with the preference of the light intensity, at any hour of the day. The data is recorded in the php My Admin is a free software tool written in php used to handle the administration of MySQL as a database. In the database the major attributes are Name, serial number, date, intensity number as a user input, date, start time, end time. XAMPP provides the local server that connects all the things bagged with WIFI, so that one can access the database by mobile PC, windows MAC IOS etc and monitor its utilization of energy consumed and saved. The data will be pilled of every hour with the change of time and intensity as well. But the end of the day, month and year that is, January, February - December. A comprehensive and cumulative analysis can be made in contrast to light used and saved plus the energy as well.

\section{Schematic diagram explanation:}

Basically! The implementation of my proposed model revolves around sensors to detect human presence, NODE-MCU (it has its layers from the Elva based firmware version, which contains the ESP8266 WIFI SOC from the following Espressif. The basis of the code repository pertains to the $\mathrm{C}$ embedded language. NODEMCU that serves as a Open source rostrum to edit/refine/construct/build. The NODEMCU has a WIFI module with numerous input/output pins containing analog and digit that serves as a serial interrelation parameter. The software corner, coding is done in c-embedded language in Arduino IDE. That is, Arduino development environment, it provides us a platform to perform basic coding and easily tackle debugging in order to code for simple applications, thus Arduino IDE is massively fabricated. On installing Arduino ide under respective operating system be it Linux, windows, mac os etc. Draft the following code of the model on the Arduino ide.

\section{Hardware subjected assembly:}

Connect the NODEMCU, with the relay amalgamate the connections via jumper wires with the Pir sensor. Itsy-bitsy details - PIR sensor contains 3 pins, ground pin (GND), output pin (DOUT), the VCC (signal). Negative terminal of PIR sensor 1 is connected to GND. DOUT (high/low) output pin of PIR sensor 1 is connected to D0 of NODEMCU. VCC that is, input voltage of PIR sensor 1 is connected to $5 \mathrm{~V}$, further which, Negative terminal of PIR sensor 2 is connected to GND. DOUT (high/low) output pin of PIR sensor 2 is connected to D1 of NODEMCU. VCC that is, input voltage of PIR sensor 2 is connected to $5 \mathrm{~V}$. The relay having 3 terminals, $1^{\text {st }}$ pin $(\mathrm{NC})$ is connected to the $5 \mathrm{~V}, 2^{\text {nd }}$ pin $(\mathrm{C})$ is connected to the D4 of the NODEMCU, $3^{\text {rd }}$ pin (NO) is connected to the GND. 


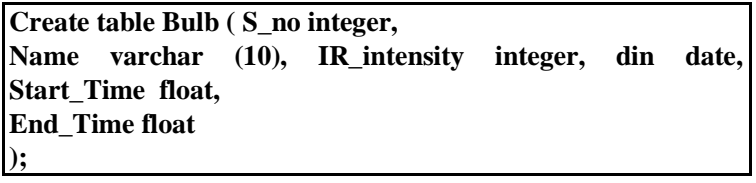

Fig.3(a) SQL commands for creation of table.

Structured query language: A data is an amalgamation of facts, objects information, further a database is a systematically oriented collection of data (unprocessed, unrefined), data in the database is easy and quick, a data management is easy in SQL as well with the creation of tables, tuples, attributes, insertion, deletion, update, modify etc. In the phpMyAdmin a database of a bulb is made with attributes such as s.no, name, intensity number, start time, end time, date. A cumulative and comprehensive analysis was made on the intensity logged in by the user. Along with the date and changed time period was noted of the intensity in a day and the month, followed by the whole entire year. In the above picture, attributes along with their data types was penned down. Next part was the insertion in each and every tuple with the respective attributes. The triagenda was to showcase the amount of energy consumed and saved by the user under the automatic lightening system via smart home automation with the shadows of IOT.

\begin{tabular}{|l|l|l|l|l|l|}
\hline $\begin{array}{l}\text { S. } \\
\text { No. }\end{array}$ & Name & IRINT & Date & $\begin{array}{l}\text { Start } \\
\text { Time }\end{array}$ & $\begin{array}{l}\text { End } \\
\text { Time }\end{array}$ \\
\hline 1 & Lanoo & 3 & $1-1-2020$ & $12: 00$ & $6: 45$ \\
\hline 2 & Lanoo & 5 & $1-1-2020$ & $6: 45$ & $10: 00$ \\
\hline 3 & Devika & 2 & $1-1-2020$ & $10: 00$ & $15: 00$ \\
\hline 4 & Chetna & 5 & $1-1-2020$ & $15: 00$ & $17: 50$ \\
\hline 5 & Lanoo & 4 & $1-1-2020$ & $17: 50$ & $19: 00$ \\
\hline 6 & Devika & 4 & $1-1-2020$ & $19: 00$ & $21: 00$ \\
\hline 7 & Chetan & 6 & $1-1-2020$ & $21: 00$ & $24: 00$ \\
\hline 8 & Jyoti & 4 & $2-1-2020$ & $12: 00$ & $8: 10$ \\
\hline 9 & Karan & 5 & $2-1-2020$ & $8: 10$ & $10: 00$ \\
\hline
\end{tabular}

Fig.3(b) The picture displays table creation of database on php "My Admin".

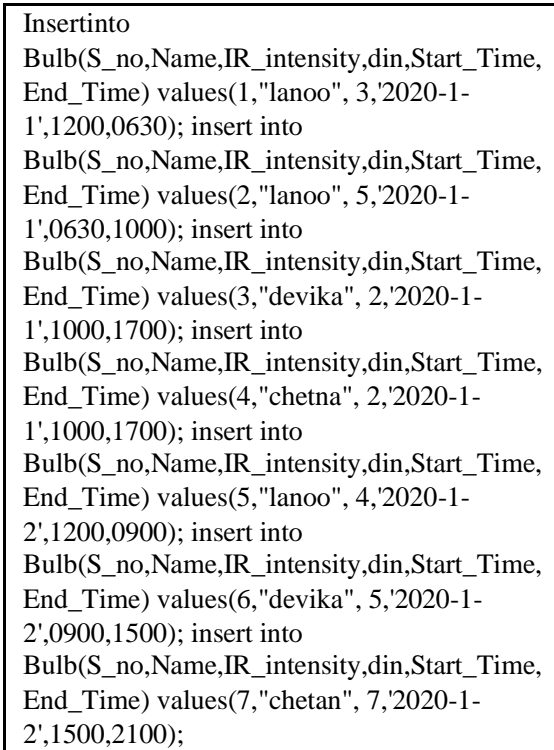

Fig.4 Insertion of the data in the database of the intensity of light per day.

\section{CONCLUSION}

We aspire and intend to live a better and patterned life. This technology IOT and automatic lightening system in the smart home automation as the project is titled to experience "IOT" by the gateway of smart home spectrum. My project is riveted on the "ON" and "OFF" of lights, with the sensors (PIR) that detects the human existence in the domestic. A key feature is the intensity that can be set by the user according to his/her will and wish. Moreover! The database prepared would serve as an enlightenment to the usage of electricity that is, the rate pattern of consumption and savings. By using this, it'll not just create conscious awareness, but it'll make the user to reduce the electricity bill and main agenda is to minimal the human intervention. Basically, with the thesis of my code.

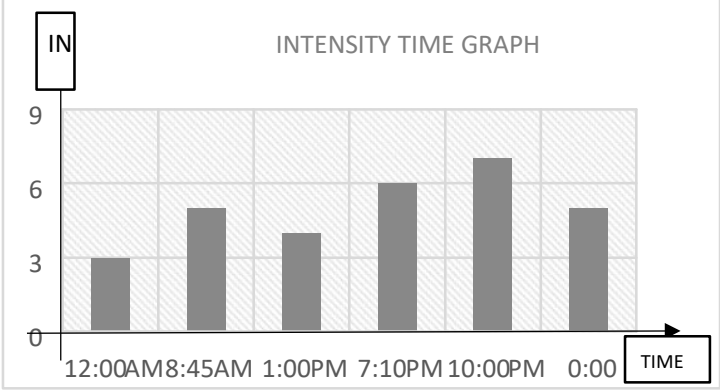

Fig.5 Shows the graph plotted between the intensity of light and the time period of a day from database showcasing the consumption of light at various intervals of time.

AC supply, if we have $10 \mathrm{~W}$ CFL-BULB bagged with the voltage dimmer, as per the definition of a voltage dimmer it'll minimal the level of the illuminance but it'll work on $10 \mathrm{~W}$ only. Hence, it won't reduce the power consumption. Contracting to it, is an LED bulb $50 \mathrm{~W}$ which works on DC supply, bagged with the voltage dimmer. Thus, it will ensure less power is consumed. Switching the humanity to the LED technology, it'll have the variation in brightness and consume less power contracting the use of the CFL's with more power equivalent to $10 \mathrm{~W}$. Also, the future work induced for the above could pertain to Working of the LED lights, so that more and more mankind could shift themselves to led and be a part of the great internet of things revolution under smart home automation.

\section{FUTURE WORK}

Further which, the smart assistance, google homes, Alexa could be used to enhance the working of the model that is without the use of the keypad 
and via a facial or speech recognition feature could work upon and it would remove the human intervention to an extent. The intensity could be lowered or highered depending upon mood and wish of the user. No indulgence of keypad would be required. For example, my project could be taken to another level that is, the human will have to say google homes set intensity 5, the intensity automatically with the speech of the user will set upon and the work will be done with the snap magic of one's voice. Further which, it'll serve as an extension to my project under future work implementation.

\section{ACKNOWLEDGEMENT}

I'm sincerely thankful to Amity University, Noida for providing me with the opportunity to write a research paper on the topic "Experience Internet of Things by the Gateway of Smart Home Spectrum" I'm utterly grateful and thankful to the authors under the reference section so as to serve as an initiation to make the paper meaningful and interesting.

I'm also thankful to the librarian and the computer lab in charge of Amity University, Noida who have helped me during the course of this research paper in different ways.

Through the research paper I have learnt a lot about the perspicacity of internet of things and encircling smart home spectrum. It has helped greatly for my analysis of the research paper.

\section{REFERENCES}

[1] Miranda, Javier, et al. "From the Internet of Things to the Internet of People." IEEE Internet Computing 19.2 (2015): 40-47.

[2] Karimi, Kaivan, and Gary Atkinson. "What the Internet of Things (IoT) needs to become a reality." White Paper, Free Scale and ARM (2013):116.

[3] Ehsani, Farzad, Silke Maren Witt-Ehsani, and Walter Rolandi. "Smart home automation systems and methods." U.S. Patent No. 9,614,690. 4 Apr. (2017). 